\title{
Escepticismo, falibilismo y verosimilitud
}

\author{
Scepticism, fallibilism, and verisimilitude
}

\author{
ILKKA NIINILUOTO \\ Universidad de Helsinki
}

Recibido: 01/09/98 Aceptado:10/09/98

Traducción: Antonio Diéguez y

Pascual Martínez-Freire

«Si se nos ocurre una idea que parece guardar una similitud con la verdad, el espíritu se llena del placer más humanizador».

Cicerón: Académica (45 a. C.)

\begin{abstract}
RESUMEN
En la epistemología moderna, el falibilismo es una vía media entre el dogmatismo y el escepticismo. Su origen histórico se encuentra en una rama de la antigua escuela del escepticismo académico. Ya que la diferencia entre las formas fuerte y débil del falibilismo, así como la distinción entre probabilidad epistémica y verosimilitud, sólo han sido comprendidas en las últimas dos décadas, no podemos esperar encontrar formulaciones claras de dichas doctrinas entre los filósofos griegos y romanos. Pero hemos mostrado que las dos ideas centrales del falibilismo -el grado de certeza y el grado de cercanía a la verdad- están presentes en la tradición escéptica al menos desde los tiempos de Cicerón.
\end{abstract}

PALABRAS CLAVE

FALIBILISMO EPISTÉMICO, HISTORIA DEL ESCEPTICISMO, VARIEDADES

DEL ESCEPTICISMO, VEROSIMILITUD.

(C) Contrastes. Revista Internacional de Filosofía, vol. XXV Nº3 (2020), pp. 115-142. ISSN: 1136-4076

Departamento de Filosofía, Universidad de Málaga, Facultad de Filosofía y Letras

Campus de Teatinos, E-29071 Málaga (España) 


\begin{abstract}
In modern epistemology, fallibilism is a middle way between dogmatism and skepticism. Its historical origin is found in a branch of the old school of academic skepticism. Since the difference between the strong and weak forms of fallibilism, as well as the distinction between epistemic probability and verisimilitude, have only been understood in the last two decades, we cannot expect to find clear formulations of such doctrines between the Greek and Roman philosophers. But we will show that the two central ideas of fallibilism -the degree of certainty and the degree of closeness to the truth- have been present in the skeptical tradition at least since the time of Cicero.
\end{abstract}

KEYWORDS

EPISTEMIC FALLIBILISM, HISTORY OF SKEPTICISM, VARIETIES OF SKEPTICISM, VERISIMILITUDE

\title{
I. INTRODUCCIÓN
}

ESTE TRABAJO INTENTA ILUSTRAR la interacción entre la filosofía sistemática y la historia de la filosofía en el caso especial del concepto de verosimilitud. Dicha noción, tras el derrumbamiento de la definición que le dio Karl Popper en 1960, ha sido el foco de un intenso trabajo lógico desde la mitad de la década de los 70, pero fue introducida por primera vez en la filosofía por la escuela escéptica ya en la época helenística.

La historia de la filosofía ha sido siempre una fuente importante de problemas y temas para la investigación filosófica sistemática. Y a la inversa, la filosofía sistemática puede tener un impacto positivo en el estudio histórico de la filosofía, especialmente en el género que Rorty (1984) llama «reconstrucción racional». El conocimiento de los desarrollos y distinciones conceptuales contemporáneos permite al historiador proporcionar, en nuestros términos, explicaciones más detalladas de las antiguas doctrinas filosóficas: puede plantear nuevas preguntas, detectar la estructura de los argumentos, localizar premisas ocultas, y buscar cosas que los estudiosos anteriores ni siquiera entendieron lo suficiente como para investigarlas. ${ }^{1}$

Por otra parte, la filosofía contemporánea puede también ejercer una influencia negativa o limitativa sobre la investigación histórica, incluso en lo que Rorty llama «reconstrucción histórica», en la cual se describe a los pensadores ya muertos «en sus propios términos». Joseph Agassi (1963) ha argumentado que la historiografía de la ciencia está normalmente cargada de suposiciones procedentes de la filosofía de la ciencia: no hay descripción pura sin alguna interpretación. Esta carga teórica puede manifestarse como

1 Un buen ejemplo lo constituye el estudio del método axiomático en la ciencia y la matemática griegas y la evaluación de sus logros y sus limitaciones a la luz de las concepciones modernas. Ver Suppes (1981). 
una tendencia a proyectar los propios puntos de vista sobre los pensadores del pasado: un positivista encuentra precursores del positivismo, un instrumentalista anticipaciones del instrumentalismo, etc. ${ }^{2}$ De manera más general, las teorías actuales pueden limitar la perspectiva del historiador a un número fijo de posiciones alternativas posibles, y forzar con ello a los pensadores antiguos a encajar dentro de ese marco. En tales casos, un progreso en filosofía sistemática puede tener un efecto liberador sobre el ámbito de las interpretaciones históricas viables.

Mi hipótesis de trabajo en este artículo es que algo así ha sucedido en la historia de la epistemología. Antes de los años sesenta el concepto de verosimilitud solía interpretarse como probabilidad, y la historia de la tradición escéptica había sido escrita desde el punto de vista de las teorías probabilistas del conocimiento y de la inferencia. Sin embargo, la larga y conocida historia de este concepto aparece bajo una nueva luz si nos acercamos a él a través de las recientes explicaciones no-probabilistas de la verosimilitud. ${ }^{3}$

\section{EPISTEMOLOGÍA Y AGNOIOLOGÍA}

El término epistemología fue introducido por James F. Ferrier en 1854. Mientras que este término ha llegado a ser normal, otro término sugerido por Ferrier, agnoiología (el estudio de la ignorancia humana), ha recibido menos atención. En esto cabe ver una indicación de las dificultades para situar las corrientes escépticas dentro de la narrativa histórica acerca de las teorías del conocimiento humano.

Como señaló John Dewey en 1929, la epistemología ha estado dominada por la «búsqueda de la certeza» (cf. Dewey, 1960). Para Platón, el conocimiento genuino (en griego, episteme) es la creencia verdadera con una explicación o una justificación. Para Aristóteles, el conocimiento científico (en latín, scientia) es necesariamente verdadero y demostrado por sus causas. Según los estoicos, el «hombre sabio» tiene impresiones verdaderas (gr. phantasiai

2 Véase, por ejemplo, la obra, en muchos sentidos excelente, de Pierre Duhem (1969) sobre la historia del debate del realismo contra el instrumentalismo. Duhem tiene la tendencia a clasificar entre los instrumentalistas (posición por la que toma partido y según la cual los enunciados teóricos no tienen valores de verdad) a aquellos realistas críticos que entienden las teorías como falsas pero cercanas a la verdad (cf. Niiniluoto, 1997).

3 Yo comencé mi propio estudio lógico de la verosimilitud en 1974 (ver Niiniluoto, 1977). El Dr. Lilli Alanen me hizo notar que San Agustín discutía en Contra Academicos el concepto de «lo-que-es-como-la-verdad». El capítulo 5 de mi Truthlikeness (1987) esboza la historia de este concepto, pero en ese momento no había leído aún a Cicerón. La primera versión del presente trabajo fue expuesta en un simposium sobre la filosofía helenística celebrado en Helsinki en Agosto de 1994. 
katakeptikai) que no pueden ser falsas. Para Tomás de Aquino, errar es un pecado. Para Descartes, la razón humana es capaz de alcanzar verdades indudables a partir de las ideas claras y distintas. Para Francis Bacon, los cánones de la inducción eliminativa garantizan la certeza de las conclusiones. Para Inmanuel Kant, hay verdades sintéticas a priori sobre la constitución del mundo fenoménico. Para muchos empiristas modernos, nuestro sistema de conocimiento descansa sobre verdades perceptivas incorregibles acerca de los datos de los sentidos. Y para los seguidores fenomenológicos de la tradición cartesiana, hay proposiciones fundamentales que son verdades evidentes por sí mismas (cf. Chisholm, 1977).

A la tradición infalibilista, que comienza con Platón, la historia standard de la epistemología añade otra corriente principal. Ésta afirma que, al menos fuera del ámbito de la lógica y la matemática, nunca tenemos razones concluyentes o una justificación absoluta para las afirmaciones fácticas, de modo que, a lo sumo, el conocimiento es probable. Charles S. Peirce acuñó en la década de 1890 para esta postura el término falibilismo (del latín fallere $=$ equivocarse, errar).

El avance de la ciencia moderna, con sus métodos empíricos y experimentales, dio origen a la idea de que hasta las mejores partes del conocimiento humano son inciertas. ${ }^{4}$ Las observaciones repetidas pueden incrementar nuestra confianza en la verdad de las hipótesis científicas, pero semejante inferencia inductiva nunca consigue la plena certeza. David Hume, en el siglo XVIII, desafió la validez de la inducción, pero la escuela empirista en filosofía de la ciencia le respondió desarrollando métodos para calcular las probabilidades de las hipótesis en relación con la evidencia empírica. De este modo, el probabilismo se convirtió en la forma más significativa de falibilismo. ${ }^{5}$

El cálculo de probabilidades fue creado por Blaise Pascal en la década de 1660 y fue desarrollado por G. W. Leibniz, el obispo Thomas Bayes y el marqués de Laplace. ${ }^{6}$ Según la escuela bayesiana, continuada en el siglo XIX por Stanley Jevons, la probabilidad es un concepto epistémico que expresa el grado de creencia en la verdad de una hipótesis sobre la base de la evidencia. John Maynard Keynes sostuvo en la década de 1920 que los grados de creencia

4 Van Leeuwen (1970) trata la emergencia de esta idea en el siglo XVI. Cf. Laudan (1981), cap. 4.

5 El término 'probabilismo' entendido en este sentido no debe ser confundido con su uso en ética, aun cuando ambas ideas no estén desconectadas. Ver Kantola (1994).

6 La exposición de Hacking (1975) subraya que, desde el surgimiento de la probabilidad a mediados del siglo diecisiete, este concepto estuvo asociado con los grados epistémicos de creencia y con las frecuencias estadísticas. Garber y Zabell (1979) sostienen que éste era un rasgo característico de los conceptos medievales de probabilidad y evidencia. 
racionales únicamente se establecen como grados de implicación parcial. Esta idea de la teoría de la confirmación fue continuada en los años cuarenta por la lógica inductiva de Rudolf Carnap y extendida a la generalización inductiva por Jaakko Hintikka en los años 60 (cf. Niiniluoto y Tuomela, 1973). En los años 20 Frank Ramsey y Bruno de Finetti consideraron que los grados de creencia eran mensurables mediante las proporciones con las que un agente está dispuesto a apostar en favor de una hipótesis. Isaac Levi (1967) desarrolló reglas inductivas de aceptación y mostró cómo puede tratar un bayesiano la idea pragmatista (Peirce, William James) de que la tarea de la epistemología es analizar los métodos para la revisión de sistemas de creencias. De este modo, el falibilismo probabilista quedó también ligado a las epistemologías anti-fundacionalistas o coherentistas. ${ }^{7}$

La interpretación frecuentista de John Venn y Peirce en la década de 1860 proporcionó otra formulación de la idea de conocimiento probable. Para Peirce, la probabilidad de un argumento inductivo es una frecuenciade-verdad (truth-frecuency) que nos dice con qué frecuencia el argumento conduce desde premisas verdaderas a una conclusión verdadera ( $C P$ 2.652). (Así, la probabilidad de una implicación deductiva sería uno). Este concepto de probabilidad fue incorporado por la lógica de la probabilidad frecuentista de Hans Reichenbach (en los años 30), en la teoría Neyman-Pearson de la inferencia estadística (en los años 40), y por la definición de conocimiento que ofreció Alvin Goldman (en los años 60) a partir de las nociones de métodos y fuentes «fiables» (reliable).

Pero esta imagen con dos ramas principales (infalibilismo y falibilismo probabilista) aún deja fuera partes importantes de la historia real.

Para empezar, Aristóteles, en los Tópicos y en la Retórica, estudió los argumentos dialécticos, en los cuales las premisas son opiniones probables, no conocidas como verdaderas sino sólo generalmente aceptadas, y las conclusiones son doxa en el sentido platónico. En la filosofía medieval, la noción de probabilidad era asignada a las creencias (lat. opinio) considerando el número de autoridades que las aprobaban (ver Byrne, 1968; Kantola, 1994). Los humanistas del Renacimiento continuaron esta tradición dialéctica (ver Jardine, 1983). Fue desarrollada de una forma nueva por G. W. F. Hegel y los seguidores de su epistemología dinámica, quienes acentuaron de diferentes maneras la idea de que la verdad y el conocimiento son los fines o límites últimos de la investigación. En la década de 1870, Peirce caracterizó la verdad como el límite hacia el cual convergería la opinión de la comunidad científica en un proceso indefinidamente largo de investigación mediante el método científico. F. H. Bradley (1893) desarrolló una explicación de los «grados de

7 Véase Pappas y Swain (1978), Lehrer (1990). 
verdad y falsedad» sobre la base de la teoría de la verdad como coherencia. Karl Marx, Friedrich Engel y V. I. Lenin consideraron la verdad absoluta como el límite al que cabe acercarse a través de una secuencia de verdades relativas o incompletas. Estas ideas hegelianas recibieron duras críticas (cf. Ewing, 1934; Quine, 1960), y los intentos de reavivarlas por parte de Wilfrid Sellars (1968) (la verdad como afirmabilidad relativa al marco conceptual ideal del que habla Peirce) y de Nicholas Rescher (1973) (la teoría de la verdad como coherencia) no han tenido mucho éxito.

En segundo lugar, ¿dónde ubicamos el movimiento escéptico? Para aquellos que piensan que el escepticismo fue una posición oscura, ya refutada con facilidad por Platón sobre la base de su incoherencia interna, puede resultar difícil entender cómo pudo sobrevivir la antigua escuela escéptica durante varios siglos, desde Pirrón de Elis (c. 300 a. C.) hasta Sexto Empírico (c. 200 d. C), y en la Academia platónica desde el siglo tercero hasta el siglo primero antes de Cristo (Arcesilao, Carnéades, Clitómaco, Filón). También resulta un tanto sorprendente saber que el escepticismo pirroniano que renació en la Francia del siglo XVI (Michel de Montaigne) usó primero su arsenal de argumentos como «una máquina de guerra» contra la nueva ciencia y el individualismo protestante, y en favor del fideísmo católico (Popkin, 1979).

Por lo general, las historias del escepticismo no van más allá de Hume y Kant. Myles Burnyeat (1983, p. 3) sugiere incluso que «Kant llevó a su final la tradición escéptica». Claro está que el escepticismo en sus formas global (e. g. Unger, 1975) y local (cf. Pappas y Swain, 1978) tuvo defensores más tardíos, pero, según Burnyeat, éstos son «una creación libre de la imaginación filosófica moderna» y «ya no descienden del antiguo linaje de Pirrón y la Academia».

En contraste con la opinión de Burnyeat, existen algunos intentos de conectar la tradición escéptica con la corriente principal del empirismo probabilista. Al comienzo del siglo XVII Marin Mersenne y Pierre Gassendi combinaron el escepticismo acerca de la metafísica con la confianza en la ciencia empírica, en la medida en que la ciencia proporciona información probable sobre las apariencias y realiza predicciones sobre el curso futuro de la experiencia. Richard Popkin (1979) asocia esta posición de «escepticismo constructivo» o «mitigado» con la posterior «concepción pragmáticopositivista del conocimiento». ${ }^{8}$

Otro argumento bien conocido extiende la cadena desde los escépticos hasta los probabilistas modernos, pasando por Carnéades y Cicerón. Muchos libros sobre la teoría de la probabilidad recuerdan que no fue sólo el obispo

8 Véase, por ejemplo, la formulación de Bas van Fraassen (1980) del «empirismo constructivo»». 
Butler en 1736, sino también Cicerón, quien afirmara que «la probabilidad es la auténtica guía de la vida». El concepto de pithanon que usa Carnéades se entiende a menudo como «probabilidad», y su «teoría de la verificación» y su «probabilismo» son considerados como «similares en cierto grado» al pragmatismo y positivismo de nuestros días (Popkin, 1979, p. xiv). Richard Jeffrey (1984) traza una línea desde Carnéades hasta de Finetti, Ramsey y Carnap.

En tercer lugar, hay aún otra pieza que puede trastocar el cuadro dibujado anteriormente. La campaña anti-inductivista de Karl Popper, comenzada con su Logik der Forschung en 1934, niega que alguna vez podamos probar que las «conjeturas» o hipótesis científicas (enunciados básicos singulares o teorías universales) sean verdaderas o siquiera probables. Este ataque al probabilismo, y la consiguiente controversia Popper-Carnap, ha alimentado la sospecha de que la posición popperiana es una forma de escepticismo. De hecho, está claro que Popper se ve a sí mismo como continuador del análisis escéptico de Hume sobre la inducción. Sin embargo, en 1960 Popper propuso una definición de un concepto comparativo de verosimilitud (truthlikeness or verisimilitude), y sostuvo que la ciencia progresa encontrando teorías cada vez más verosímiles (Popper, 1963, 1979). Al mismo tiempo, Popper defendió el realismo científico frente al instrumentalismo y las formas más estrechas de empirismo: las teorías han de ser entendidas de modo realista, como conjeturas sobre entidades y procesos no observables directamente en el mundo. Popper se calificó a sí mismo como un falibilista (Popper, 1963, p. 228), sin embargo, su filosofía difiere claramente de las versiones empirista y probabilista de esta doctrina.

Popper no relacionó en detalle sus tesis falsacionistas con el escepticismo antiguo. De entre los filósofos griegos, sólo estudia brevemente a Jenófanes y a Sexto Empírico como precursores de su «racionalismo crítico». A Cicerón lo menciona una vez como el filósofo que introdujo la 'probabilidad' y la 'verosimilitud' en sentido subjetivo. ${ }^{9}$

9 Véase Popper (1963), pp. 236, 404. Popper atribuye a Jenófanes la tesis de que «buscamos la verdad, pero no podemos saber cuándo la hemos encontrado» (ibid. p. 226). Sostiene que Jenófanes usó el término eoikota en un sentido objetivo para expresar «similitud con la verdad», sin embargo, los fragmentos son escasamente concluyentes. Feyerabend (1984) también discute la filosofía de Jenófanes, pero traduce aquel término como «plausible» o «semejante-a-laverdad» (truth-seeming). En dos libros escritos en la tradición popperiana que llevan la palabra 'escepticismo' en el título, no se hace ninguna comparación con el escepticismo de la Academia (ver Watkins, 1984; Musgrave, 1993). Musgrave contrasta su realismo falibilista débil y noprobabilista con el escepticismo pirrónico. Por otra parte, no he visto ni un solo estudioso del escepticismo antiguo que se refiera a Popper o a los debates recientes sobre la verosimilitud. 
Hay una importante tradición de realismo falibilista en filosofía de la ciencia que comienza en el siglo XVII con la consideración del método de hipótesis. Robert Boyle, en su The Sceptical Chymist de 1661, usó a Carnéades como portavoz de un escepticismo capaz de poner en tela de juicio las teorías aristotélica y paracelsiana de la materia. Boyle, que era un defensor del corpuscularismo, dejaba lugar a que «el entendimiento formara una hipótesis», y argumentaba que la ciencia realiza una aproximación gradual a la verdad vía posiciones falsas intermedias. ${ }^{10} \mathrm{El}$ progreso científico fue visto como análogo a los métodos de iteración y aproximación para resolver ecuaciones en matemáticas. Como señaló Laudan (1973), la caracterización de Peirce de la naturaleza auto-correctora de la ciencia pertenece a esta tradición, así como la vindicación de la inducción por parte de Reichenbach y la noción popperiana de verosimilitud (cf. Laudan, 1981). Opiniones similares sobre la aproximación a la verdad, que Laudan no menciona, expresaron los idealistas británicos (Bradley) y los filósofos marxistas (Engels), motivados por la metafísica evolutiva hegeliana (cf. Niiniluoto, 1977, 1978).

Estas observaciones nos invitan a reconsiderar las similitudes entre los falibilistas contemporáneos y los antiguos escépticos. Sin sugerir que exista alguna influencia causal real, tiene interés preguntarse quiénes son hoy en día los auténticos herederos del escepticismo pirrónico y académico. Podemos también albergar la esperanza de que, al responder a esta pregunta, consigamos descubrir algunos aspectos hasta el momento inadvertidos de las doctrinas de los filósofos antiguos.

\section{FALIBILISMO DÉBIL Y FALIBILISMO FUERTE}

Con el propósito de la comparación histórica es útil hacer algunas distinciones epistemológicas sistemáticas.

Peirce definió el falibilismo como la doctrina según la cual «la gente no puede alcanzar una certeza absoluta sobre las cuestiones de hecho» ( $C P$ 1.149). Peirce añadía ( $C P$ 1.137) que «es una verdad antigua que en la ciencia no podemos estar seguros de nada», una verdad enseñada ya en la Academia de Platón. Por tanto, «cualquier proposición científica es siempre susceptible

10 Cf. Laudan (1981), p. 42. En tanto que defensor temprano del método hipotéticodeductivo, Boyle es un realista crítico, y creo que es desorientador caracterizarlo como un «escéptico constructivo» en el sentido de Popkin (ver van Leeuwen, 1970, p. 105). 
de ser refutada y rápidamente abandonada» ( $C P 1.120)$. Esto no significa, sin embargo, que la verdad y el conocimiento sobre la realidad sean inaccesibles:

Nada hay, pues, que nos impida conocer las cosas externas como realmente son, y lo más probable es que así las conozcamos en un sinnúmero de casos, aunque nunca podamos estar absolutamente ciertos de haberlo hecho en un caso especial. $(C P 5.311)$

Estas frases formulan lo que cabe llamar falibilismo en sentido débil (cf. Niiniluoto, 1978): nuestras creencias pueden ser falsas, dado que nunca poseemos una justificación concluyente de su verdad. El conocimiento humano es siempre incierto y corregible, pero aun así en algunos casos es verdadero, aunque no podamos estar seguros de ello.

Según la formulación bayesiana del falibilismo débil, el grado de incertidumbre de una proposición puede ser expresado por su probabilidad epistémica: $\mathrm{P}(\mathrm{h} / \mathrm{e})$ es el grado de creencia racional en la verdad de la hipótesis h sobre la base de la evidencia e. El valor máximo de esta probabilidad es igual a la certeza:

(1) $\mathrm{P}(\mathrm{h} / \mathrm{e})=1$ si y sólo si h es cierta sobre la base de e.

El valor mínimo $\mathrm{P}(\mathrm{h} / \mathrm{e})=0$ corresponde al caso en el que la falsedad de $\mathrm{h}$ es cierta sobre la base de e.

Para la formulación frecuentista del falibilismo débil, en cambio, una hipótesis ha de ser tratada como un miembro aleatorio del conjunto de conclusiones similares obtenidas directamente de una fuente (como la percepción) o por un método de inferencia (como una prueba estadística). Esta es la definición no-bayesiana que da Peirce de la «probabilidad de la inducción». La probabilidad de una hipótesis h es su frecuencia-de-verdad con respecto al método $\mathrm{M}$, es decir, la frecuencia relativa con la que el método $\mathrm{M}$ es capaz de generar conclusiones verdaderas. Por tanto, la probabilidad de $\mathrm{h}$ alcanza el valor máximo de uno si y sólo si h ha sido obtenida mediante un método que sea completamente fiable (cf. Goldman, 1984), esto es, mediante un método que genere invariablemente conclusiones verdaderas.

Algunas de las declaraciones de Popper sugieren que él tenía como objetivo conseguir una versión no-probabilista del falibilismo débil. Cuando una hipótesis audaz sale bien parada de pruebas severas, adquiere un grado positivo de corroboración, pero, a diferencia de los grados de confirmación de los bayesianos, la corroboración no es una probabilidad (aunque pueda ser una función de probabilidades). Popper, como admirador de la teoría tarskiana de la verdad como correspondencia, no negaba la posibilidad de que las teorías científicas fuesen verdaderas. Pero solía afirmar que todas las teorías serán falsadas, y «la mayoría de las veces» podemos errar el blanco de la verdad 
«por un amplio margen» (Popper, 1963, p. 16).

También Peirce usaba ocasionalmente frases que sugieren que la verdad era para él sólo «un límite ideal hacia el que tendería una investigación sin final para producir creencia científica» (CP 5.565). En particular, la validez de un argumento inductivo consiste en el hecho de que «sigue un método tal que, si se persiste en él debidamente, ha de conducir a largo plazo, por la propia naturaleza de las cosas, a un resultado que se aproxima indefinidamente a la verdad» (CP 2.781) Así, una sucesión típica de hipótesis científicas es una secuencia de teorías falsas $h_{1}, h_{2}, \ldots$, pero una secuencia que converge hacia una teoría verdadera $\mathrm{T}$ ( $\sin$ alcanzarla nunca en un número finito de pasos). De la misma manera, Popper sostenía que el progreso científico está constituido por secuencias de teorías falsas en las que la teoría posterior mejora a la anterior por estar «más cerca de la verdad».

Esta idea nos muestra una posición a la que podemos llamar falibilismo en sentido fuerte (cf. Niiniluoto, 1978): los errores no sólo son posibles, sino reales; nuestras creencias son falsas, aunque pueden estar «cerca de la verdad» o ser «aproximaciones a la verdad».

Una formulación temprana del falibilismo fuerte la encontramos en 1440, en el De docta ignorantia de Nicolás de Cusa. Éste utilizó un sugerente modelo geométrico para ilustrar cómo nuestro conocimiento finito puede aproximarse indefinidamente a la verdad infinita. La relación de nuestro intelecto con la verdad es como la del polígono con el círculo: multiplicando el número de ángulos el polígono se aproximará al círculo sin llegar a identificarse con él. En su doctrina de la «docta ignorancia», Nicolás de Cusa abogó por la teología negativa: Dios es inefable, pero podemos acercarnos a la verdad sobre Él usando proposiciones negativas.

Los metafísicos hegelianos fracasaron a la hora de dar sentido a la idea del acercamiento a la verdad (cf. Niiniluoto, 1987, cap. 5). Entre los problemas que provocaron este fracaso está el que Bradley fuera influido por la definición hegeliana de la verdad como coincidencia de un objeto con su concepto o esencia. Un juicio como 'esta rosa es roja' posee, según Bradley, cierto «grado de falsedad», porque no dice todo acerca del objeto, por ejemplo, que la rosa tiene hojas verdes. Sin embargo, esto significa confundir la falsedad (información incorrecta) con la verdad incompleta (cf. Ewing, 1934). Y ambigüedades parecidas se pueden encontrar en los textos de los seguidores marxistas de Hegel.

La definición popperiana de verosimilitud de 1960 (ver Popper, 1963) tiene la virtud de especificar unívocamente como meta la verdad total, es decir, la clase completa $\mathrm{T}$ de las verdades (en un lenguaje dado L). El contenido de verdad de una teoría A (en cuanto una clase de enunciados en $\mathrm{L}$ deductivamente cerrada) es la intersección de A y T, y el contenido de 
falsedad de A es su intersección con el complemento -T de T. De este modo, el contenido de falsedad de A contiene todas las consecuencias falsas de A; las cuales pueden denominarse errores de falsedad de A (cf. Niiniluoto, 1987, p. 159). Una teoría verdadera tiene un contenido de falsedad vacío, pero el tamaño de su contenido de verdad depende de la informatividad de A. La diferencia $\mathrm{T}-\mathrm{A}$ entre $\mathrm{T}$ y $\mathrm{A}$ contiene aquellos enunciados verdaderos que $\mathrm{A}$ no consigue implicar; los cuales son, por tanto, los errores de ignorancia de A. Dicho de manera intuitiva, la definición cualitativa que Popper ofrece de la verosimilitud (truthlikeness) afirma que una teoría A es verosímil (truthlike) si y sólo si su coincidencia con la verdad total $\mathrm{T}$ es relativamente amplia, es decir, A comete tan pocos errores de falsedad y errores de ignorancia como sea posible.

David Miller y Pavel Tichy probaron en 1974 que la definición de Popper no puede dar sentido a la comparación entre teoría falsas; si una teoría A es más verosímil en el sentido de Popper que una teoría $\mathrm{B}$, entonces $\mathrm{A}$ tiene que ser verdadera. El mismo año de 1974 comenzó un nuevo programa, el enfoque de la similitud (similarity approach). Expresado en forma intuitiva, este programa sostiene que una teoría (en un lenguaje dado L) es verosímil (truthlike) si los estados de cosas que permite están cerca de los verdaderos estados de cosas (tal como son especificados en L). La verdad total es explicada mediante una descripción completa $\mathrm{T}$ del estado verdadero, es decir, $\mathrm{T}$ es el enunciado verdadero más informativo en $\mathrm{L}, \mathrm{y}$ dicho enunciado T se toma como el «objetivo» de un problema cognitivo. El grado VS(h,T) de verosimilitud (verisimilitude) de un enunciado h en relación con el objetivo $\mathrm{T}$, una vez normalizado entre 0 y 1 , satisface lo siguiente:

(2) $\operatorname{VS}(h, T)=1$ si y sólo si h es la verdad total T.

En general, el grado VS(h,T) depende de la similitud o semejanza entre el contenido de h y T. Una teoría altamente verosímil debe preservar la verdad (esto es, permitir el estado $\mathrm{T}$, o al menos estados cercanos a $\mathrm{T}$ ) y excluir estados alternativos alejados de T. Dependiendo del problema cognitivo, el objetivo T puede ser, por ejemplo, un parámetro con valores reales, una distribución de individuos en un sistema de clasificación, una ley cualitativa o cuantitativa, o una teoría completa de primer orden.

Cuando lo que se exige es sólo que una teoría permita algunos estados cercanos al estado verdadero, obtenemos la noción de verdad aproximada (approximate truth). El grado $\mathrm{AT}(\mathrm{h}, \mathrm{T})$ de verdad aproximada de $\mathrm{h}$ con respecto a T tiene un valor máximo de 1 si h viene implicada por la verdad $\mathrm{T}$ : 


\section{(3) $\operatorname{AT}(h, T)=1$ si y sólo si h es verdadera.}

La noción de verosimilitud (truthlikeness) añade el requisito de que la teoría $\mathrm{h}$ debe ser informativa acerca de la verdad. Por ejemplo, una tautología es lógicamente verdadera, y por tanto es obvio que también es aproximadamente verdadera, pero no es verosímil en alto grado, puesto que comete muchos errores de ignorancia. Es más, resulta posible que una hipótesis falsa pero informativa sea más verosímil que una verdad débil.

Dos monografías, Oddie (1986) y Niiniluoto (1987), han expuesto, con algunas diferencias importantes, los detalles de este enfoque de la similitud. ${ }^{11}$ Dicho enfoque resuelve el problema suscitado por el teorema Miller-Tichy, y da respuesta a la famosa objeción de Quine (1960) contra Peirce de que la relación 'más cerca que' no está definida para teorías. Nos permite asimismo definir de una manera precisa lo que significa para una secuencia de enunciados converger hacia la verdad T, y nos ayuda así a explicar locuciones típicas de la tradición del falibilismo fuerte. Sin embargo, las aplicaciones potenciales del concepto de verosimilitud no excluyen el falibilismo débil, puesto que los problemas cognitivos incluyen normalmente como objetivo la verdad pertinente en cada caso.

Si el estado verdadero de cosas $\mathrm{T}$ es desconocido, como sucede en un problema cognitivo auténtico, resulta entonces obvio que no es posible medir real o directamente la distancia entre una teoría h y T. Por ello, el concepto de verosimilitud puede parecer puramente teórico, inventado para mostrar que tiene sentido hablar sobre la aproximación a la verdad, pero sin ninguna otra aplicación práctica. Popper (1979) sostuvo que sus grados de corroboración podrían servir como indicadores de verosimilitud, pero no aportó ninguna razón para ello. En Niiniluoto (1977) se expuso que los grados de verosimilitud pueden ser estimados mediante su valor esperado si se define sobre los estados relevantes de cosas una distribución de probabilidad epistémica racional. Si una de las hipótesis informativas resulta tener una alta probabilidad, tendrá también el grado mayor de verosimilitud esperada (expected verisimilitude), aunque en general la verosimilitud estimada (estimated truthlikeness) se diferencia de la probabilidad. Por ejemplo, una tautología es máximamente probable, pero no posee un alto grado de verosimilitud esperada. En cambio, una hipótesis refutada, cuya probabilidad, dada la evidencia, es cero, puede aún ser estimada como cercana a la verdad. Estos resultados muestran que el problema de la estimación de la verosimilitud no es ni más ni menos difícil que el tradicional problema de la inducción (cf. Niiniluoto, 1987, cap. 7).

11 Quizás la diferencia más importante consiste en el principio, aceptado por Popper y Niiniluoto y negado por Oddie, de que, entre enunciados verdaderos, una mayor fuerza lógica implica una mayor verosimilitud. 


\section{FALIBILISMO VS. ESCEPTICISMO}

Si se definiera el escepticismo como la doctrina que niega la existencia o la posibilidad del conocimiento en el sentido platónico fuerte (cf. Unger, 1975), todos los falibilistas serían escépticos, puesto que niegan la idea de una certeza completa o de una justificación concluyente. Sin embargo, el falibilismo débil no debería ser entendido como una negación de la posibilidad del conocimiento, sino más bien como una recomendación de que la noción de justificación se entienda en el sentido probabilístico.

La distinción entre escepticismo y falibilismo fuerte no está tan clara. Pero también podemos decir que hasta el falibilista fuerte admite una noción débil de justificación en términos de verosimilitud. Incluso en los casos en que se sabe que una hipótesis es falsa, ésta puede tener alguna justificación por estar más cerca de la verdad que sus rivales, y puede merecer el título de «conocimiento» si está lo suficientemente cerca de la verdad (pace Musgrave, 1993, cf. Niiniluoto, 1997). La estructura del falibilismo fuerte presupone así la noción de verdad, si bien la considera como un objetivo al que aproximarse.

Por esta razón, es natural definir el escepticismo como la posición que niega la posibilidad del conocimiento cierto y verdadero y rechaza la posibilidad de realizar progresos hacia la verdad o de hacer estimaciones sobre la cercanía a la verdad.

Paul Feyerabend (1987, p. 76) efectuó una reciente formulación del escepticismo en tal sentido: «Para todo enunciado, teoría, punto de vista, en el que se crea (como verdadero) con buenas razones, existen argumentos que muestran una alternativa en conflicto que es al menos tan buena, o incluso mejor».

Veamos ahora cómo han propuesto tratar los falibilistas contemporáneos los problemas de la preferencia cognitiva y pragmática.

En un problema cognitivo nos preguntamos cuál de las hipótesis alternativas es la mejor desde un punto de vista epistémico. ¿Qué hipótesis es más aconsejable por sus virtudes epistémicas? ¿Cuál de entre las hipótesis rivales posee mejores garantías para ser incluida en nuestra visión del mundo?

El escéptico niega que pueda haber una diferencia epistémica o evidencial significativa entre las hipótesis alternativas y, por tanto, recomienda la suspensión del juicio. En el tipo más simple de problema cognitivo - ¿Es h verdadera o no?-, el escéptico contesta 'No lo sé'; él no cree ni en h ni en $\sim \mathrm{h}$, de modo que su respuesta puede representarse formalmente como la disyunción h V $\sim$ h (cf. Levi, 1967).

Los falibilistas débiles pueden ser divididos en dos grupos. Carnap y Jeffrey argumentan que la conclusión de una inferencia inductiva nunca consiste en la aceptación de una hipótesis como verdadera, sino solo en la 
asignación de su grado de probabilidad o su grado de confirmación sobre la base de la evidencia total disponible (ver Carnap, 1971). Por lo que a la aceptación se refiere, el carnapiano coincide con el escéptico: ha aprendido de Hume que las inferencias inductivas son inciertas, y desea evitar el error. Coincidiría, pues, literalmente con el principio de W. K. Clifford, que sostiene que nunca debe creerse en nada sobre bases insuficientes. Pero, a diferencia del escéptico, este falibilista débil precavido utiliza la probabilidad posterior o el grado de confirmación para indicar una preferencia epistémica entre las hipótesis.

Levi y Hintikka, en cambio, son falibilistas débiles atrevidos que especifican las condiciones para la aceptación tentativa de hipótesis y su incorporación a nuestro cambiante y revisable cuerpo de conocimientos. Si deseamos obtener información sobre la realidad, y superar así el agnosticismo, tenemos que asumir riesgos epistémicos y, como dice Levi (1967), «apostar por la verdad».

Cabe hacer una distinción similar entre versiones precavidas y atrevidas del falibilismo fuerte. Por lo general, Popper es precavido y dice que las teorías son «aceptadas» sólo para pasar pruebas ulteriores, pero, con todo, la verosimilitud proporciona en principio un instrumento para jerarquizar teorías, y favorece a las teorías audaces e improbables con un gran contenido de verdad. Si se toma como una regla de aceptación la maximización de la verosimilitud esperada (cf. Niiniluoto, 1987, cap. 12), la estructura resultante llega a ser más atrevida que la regla de Levi, puesto que nos permite preferir algunas falsedades informativas a verdades triviales. Pero está claro que este grado de atrevimiento está todavía lejos de ser imprudente, precipitado o irresponsablemente osado, como lo es la doctrina de la voluntad-de-creer de William James (cf. James, 1956) o el fideísmo que aboga por creencias sin una base o una evidencia que las sostenga (cf. Popkin, 1979).

En un problema pragmático nos preguntamos cuál de las acciones alternativas es la mejor en una situación dada. ¿Cómo debemos elegir o decidir racionalmente entre los cursos disponibles de acción? ¿Cómo podemos usar el conocimiento empírico y teórico a modo de recurso en una deliberación práctica? ${ }^{12}$

Una regla simple a la que el falibilista débil precavido puede recurrir, y que cabe llamar la regla de la probabilidad alta, consiste en encontrar entre las hipótesis alternativas la que sea más probable y actuar como si fuese verdadera. Un falibilista débil atrevido propondría que los enunciados

12 El tratamiento que hace Aristóteles de la deliberación práctica en la Ética a Nicómaco es infalibilista, en el sentido de que no hay ninguna incertidumbre acerca de las consecuencias de las acciones alternativas. 
aceptados inductivamente sean usados como guía para una práctica exitosa (cf. Peirce CP 5.170, 5. 199). Popper (1979, p. 22) afirma que, por las razones que ya dio Hume, nunca debemos «confiar» en una teoría, pero si hemos de actuar, entonces este «problema pragmático de la inducción» debe resolverse prefiriendo como base para la acción «la teoría mejor contrastada», es decir, la teoría con el mayor grado de corroboración y de verosimilitud. A esto se le puede llamar la regla de la verosimilitud alta.

Estas propuestas no toman en consideración las utilidades y las pérdidas de las consecuencias de las acciones alternativas en relación con los diferentes estados de la naturaleza. En la teoría de la decisión bayesiana se les asigna a dichas consecuencias un valor numérico de utilidad (en relación con los fines prácticos propuestos en la situación de la toma de decisión), y la acción que tenga el grado mayor de utilidad esperada es la recomendada para la acción. El resultado puede ser diferente al de la regla simple de la probabilidad alta. En opinión de Carnap (1971), el papel del científico es trabajar como consejero de los que toman decisiones y proporcionar las probabilidades posteriores que puedan usarse para calcular los valores de la utilidad esperada. De este modo, la probabilidad (junto con las preferencias prácticas y las utilidades del que toma la decisión) es una guía para la vida.

\section{ESCEPTICISMO PIRRÓNICO Y ACADÉMICO}

Ahora ya estamos en disposición de considerar la escuela helenística del escepticismo a la luz de nuestra discusión sistemática. Existen todavía debates en curso sobre la interpretación de los motivos y las tesis de los pensadores antiguos (ver Scofield et al., 1980; Burnyeat, 1983; para los textos, ver Long y Sedley, 1988). Mi interés se centra en las diferentes posiciones esbozadas en los debates, y no tanto en si alguna figura histórica abogó realmente por una cierta opinión o simplemente la presentó en un argumento dialéctico contra sus oponentes.

Pirrón de Elis (360-270 a. C.) es citado a menudo como el fundador de la escuela escéptica. Pirrón enseñó que la ataraxia, la tranquilidad mental o un estado imperturbado y tranquilo del alma, puede alcanzarse si nos abstenemos de dar nuestro asentimiento a proposiciones que no pueden ser conocidas como ciertas o verdaderas. Enesidemo, que resucitó el pirronismo en torno al año 80 a. C., formuló diez «tropos» o modos de suspensión del juicio, que resultan de la posibilidad de cometer errores en la percepción sensible y en el razonamiento. Sexto Empírico (c. 200 d. C.) presentó como parte de la posición pirrónica la siguiente tesis: «A todo argumento se opone otro argumento igual» (Sexto Empírico, 1985, p. 35). Este equilibrio de argumentos se aplica también a la cuestión acerca de la posibilidad del conocimiento: un 
verdadero escéptico no la afirma ni niega dogmáticamente, sino que «continúa buscando». (ibid. p. 31).

Arcesilao encabezó la Academia platónica en el año 273 a. C, y con él comenzó el predominio del escepticismo académico, que duró alrededor de dos siglos. La noción central de Arcesilao es la epoche, la suspensión del juicio o la negación a asentir ante cualquier cuestión. Esto iba dirigido contra la noción estoica de katalepsis o creencia infalible, y contra la idea de que existe un criterio para distinguir las impresiones verdaderas de las falsas.

La suspensión del juicio sobre la proposición h garantiza la evitación del error de falsedad; el contenido de falsedad popperiano no se ve así incrementado. Hemos visto que ésta es la motivación de los falibilistas precavidos, quienes se niegan a asumir el riesgo de aceptar una falsedad. Pero la epoche sobre h no es un remedio contra todo error puesto que conduce a errores de ignorancia: no deja que se acepte la alternativa verdadera en $\mathrm{h}$ o $\sim \mathrm{h}$. Y si, como dijo Aristóteles, todo hombre por su naturaleza desea el conocimiento, ¿por qué debería esta ignorancia contribuir a la imperturbabilidad de nuestro espíritu? Además, ¿es realmente coherente suspender el juicio en todas las cuestiones?

Quizás la objeción más seria que recibió fue el argumento estoico de la apraxia: un hombre que no da su asentimiento a nada, no puede actuar. Este argumento tenía dos formas, la primera afirmaba que el escéptico nunca será capaz de decidir qué hacer, la segunda que el escéptico no será capaz de actuar (ver Striker, 1980). A la última objeción se contestó negando la teoría estoica de que toda acción voluntaria implica un asentimiento. Como respuesta a la primera objeción, Arcesilao tomó prestada de los estoicos la noción de eulogon para indicar aquellos fenómenos que son suficientemente «razonables» para servir como base de la acción. Su sucesor más importante, Carnéades (c. 214129 a. C.) utilizó el término pithanon para referirse a las impresiones falibles que son adecuadas para conducir la vida. De este modo, la doctrina oficial de la Academia fue que la acción y la decisión eran posibles sin asentimiento (gr. synkatathesis, lat. adsensio, adprobatio).

En la tradición de la retórica griega, pithanon era usado para expresar el carácter «persuasivo» o «convincente» del propósito final de una pieza retórica. Cicerón, en su De Inventione (81-80 a. C.), tradujo este término como probabilis, del verbo latino probare, en el sentido de «convencer a la audiencia» (ver Glucker, 1995). El término veri simile fue usado por algunos autores contemporáneos de Cicerón, como Plauto y Terencio, en correspondencia posiblemente con el término griego eikos (probable, creíble) (cf. Popper, 1963). En retórica, eikos hace referencia a los argumentos particulares, parciales y de apoyo que son más débiles que los argumentos verdaderos (gr. aletheia) o ciertos. Según Glucker (1995), Cicerón siguió coherentemente esta distinción terminológica en sus obras retóricas. 
En su Academica (45 a. C.), Cicerón expuso el pensamiento del escepticismo académico. Allí tradujo el término pithanon, empleado por Carnéades, por los términos latinos veri simile y probabile, y aplicó estos términos tanto a las doctrinas filosóficas debatidas como a las percepciones de los sentidos en tanto que guías para la vida. ${ }^{13}$ De modo que, para Cicerón, la guía para la vida es la «verosimilitud» o «probabilidad». Cuando San Agustín, cuatrocientos años más tarde, atacó a los escépticos en Contra Academicos (385 d. C.), su atención se centró en la formulación ciceroniana de la doctrina académica.

Para Sexto Empírico, resulta dogmático asentir a, o dar una opinión sobre, «algo no-evidente». La epoche ya no es una negación completa de todo asentimiento: «El escéptico, por supuesto, acepta las sensaciones que se derivan necesariamente de las impresiones de los sentidos», pero debe hablar de «lo que parece» «sin permitirse una opinión o efectuar enunciados positivos sobre la realidad de las cosas externas» (op. cit., pp. 36-37). Sexto Empírico no estaba dispuesto a aceptar los grados de credibilidad de Carnéades, que encontraba dogmáticos, pero recomendaba más directamente que debemos «vivir de acuerdo con las costumbres, las leyes y las instituciones de nuestro país, y con nuestros propios sentimientos naturales» (ibid., p. 37).

\section{INTERPRETACIONES DE CARNÉADES}

Lo que más nos interesa para nuestros propósitos es considerar las diversas interpretaciones del concepto pithanotes de Carnéades y del concepto verisimilitudo de Cicerón.

\section{VI.1. PROBABILIDAD EPISTÉMICA}

Una interpretación tradicional de pithanotes es la de probabilidad epistémica, credibilidad o plausibilidad. Por ejemplo, Copleston (1966, p. 417) dice que Carnéades «elaboró una teoría de la probabilidad», y mostró «cómo podemos aproximarnos a la verdad - aun cuando nunca nos sea posible alcanzar la certeza- acumulando razones para aceptar una opinión». Popkin (1979, p. xiv) afirma que Carnéades desarrolló «un tipo de probabilismo».

13 Para el primer uso, ver la conclusión de De natura deorum: tras discutir las doctrinas estoicas y epicúreas sobre la religión, Cicerón tiene la sensación de que el representante de la primera concepción «se acerca más a una similitud con la verdad» (ad veritatis similitudinem videretur esse propensior) (ver Cicerón, 1956, p. 383). Glucker (1995), p. 130, sostiene que Cicerón usó casi constantemente probabile en conexión con la acción práctica. Yo no encuentro esta distinción, por ejemplo, en el importante pasaje de Acad., II, xxxi. Tiendo a coincidir con Popper en que Cicerón usó los dos términos clave como sinónimos, pero no estoy convencido de que los usara sólo en un sentido subjetivo (ver Popper, 1963, p. 404). 
Para P. P. Hallie, pithanon expresa varios «grados de certeza» o «grados de plausibilidad» en tanto que «están implicadas las sucesiones de experiencias o percepciones en la vida diaria» (Sexto Empírico, 1985, p. 23). A. A. Long (1986, p. 96) afirma que «Carnéades llegó a una teoría del conocimiento que anticipa en muchos aspectos las modalidades modernas de empirismo». Jeffrey (1984) traza una línea directa desde Carnéades y Cicerón, vía Montaigne y Hume, hasta las teorías probabilistas de la inducción de de Finetti y Carnap.

Entre las impresiones que son pithane o «parecen verdaderas» Carnéades distinguió tres niveles de «persuasividad». El primero viene caracterizado por la «viveza», el segundo por ser convincentes y sin estorbos (esto es, no hay otras impresiones que las hagan implausibles), y el tercero por estar además comprobadas o escrutadas (ver Bett, 1989). Aunque dichos niveles no muestran aún ningún indicio de estructura matemática, cabe verlos como una tentativa rudimentaria dirigida hacia una teoría de los «grados de creencia» o «grados de credibilidad». En el De officiis (II, 7-8), Cicerón afirma que la «probabilidad» ha de basarse en «estimaciones comparativas de los argumentos de las dos partes». ${ }^{14}$ Las traducciones ciceronianas veri simile y probabile han fortalecido la interpretación de que nos encontramos aquí con una anticipación de la teoría posterior de la probabilidad epistémica. Bajo la influencia de Cicerón y Quintiliano, estos términos fueron usados en las teorías poéticas del siglo XII (Mehtonen, 1996) y por los retóricos humanistas del siglo XV (Jardine, 1983). Cuando se desarrolló el cálculo matemático de probabilidades en el siglo XVII, los términos probabilitas y verisimilitudo eran usados regularmente como sinónimos. ${ }^{15}$ En muchas lenguas, la palabra para «probable» (probable) (que en inglés significa «que se puede investigar» (probing), «que se puede demostrar» (proving), «que se puede argumentar» (arguing), o «que se puede someter a prueba» (testing)) se forma a partir de las palabras «verdadero» (true) y «similitud» (likeness). Tal sucede con el término sueco sannolik, en tanto que en finés todennäköinen significa «queparece-verdadero»».

Si esta interpretación probabilística va en la dirección correcta, los escépticos de la Academia son falibilistas débiles. En lo referente a los problemas de la cognición, son además falibilistas precavidos que se abstienen de asentir o aceptar.

Para los problemas prácticos de la acción, el escéptico de la Academia

14 Citado por Jardine (1983), p. 267. Para los grados de probabilidad en las discusiones medievales, véase Byrne (1968).

15 La Lógica de Kant usa «verosimilitud» (Scheinbarkeit) en un sentido epistemológico especial que se distingue del otro concepto doxástico de «probabilidad» (ver Kant, 1960, p. 89). Para usos subjetivos anteriores de este término, véase Kantola (1994). 
recomienda la «probabilidad» como canon «tanto en el modo de conducir la vida como en la investigación y la discusión filosóficas» (Cicerón, Acad. II, x). La certeza o el asentimiento no son necesarios en la actividad «de emprender un viaje, sembrar un cultivo, casarse con una mujer, engendrar una familia, ni para muchas cosas en las que no se estará siguiendo nada que no sea la probabilidad» (II, xxxiv). Cicerón no nos dice mucho sobre la estructura de la toma de decisiones, salvo en su ejemplo acerca de un hombre sabio que quiere embarcar y navegar «desde aquí hasta Puteoli»; entonces «con una tripulación en la que se pueda confiar, un buen timonel y un tiempo tranquilo, parecería probable que llegara allí a salvo» (II, xxxi). ${ }^{16}$

Este ejemplo hace pensar que Cicerón está anticipando la regla de la probabilidad alta para la acción práctica. ${ }^{17}$ Por otra parte, sería erróneo afirmar que su explicación de la acción encaja en la teoría de la decisión, ya que no hay indicación alguna de que Cicerón contemplara acciones alternativas y comparara acciones diversas en función de algo parecido a sus utilidades esperadas.

16 Juha Sihvola (1996) señala que en estos pasajes, donde Cicerón discute la interpretación de Carnéades por Clitómaco, el término para «seguir» o «aprobar» una propuesta es sequentes o adprobari mejor que el término estoico adsentior (asentir, aceptación como verdadero o cierto). Él sostiene que ya los seguidores de Carnéades entendieron de dos modos diferentes la negación de su maestro de la doctrina estoica de la aceptación fuerte (adsention): para Clitómaco, el sentido débil de aprobación no indica relación alguna de una propuesta con la verdad, la probabilidad o la verosimilitud, y para Cátulo la aprobación débil es aceptar como probable. Sihvola afirma además que esta última opinión, que condujo a Cátulo al escepticismo dogmático, estaba presupuesta en Cicerón y San Agustín. En mi opinión, es natural pensar que «seguir» una impresión pithane, o su «aceptación como probable», no se debe entender como si implicara una «aceptación» en el sentido fuerte de adsention. Pero, ya que Cicerón tradujo pithanon como probabile y veri simile, no veo cómo el seguir una impresión que tenga esa cualidad pueda separarse de cualquier planteamiento relativo a su probabilidad o a su verosimilitud, al modo en que Sihvola interpreta a Carnéades y a Clitómaco. En la discusión moderna, existe la distinción, relacionada con ésta, entre aceptar una proposición y aceptar una estimación de su probabilidad. Carnap negó que se pudieran aceptar las hipótesis como verdaderas, pero afirmó que los enunciados metalingüísticos de probabilidad en la lógica inductiva eran verdades analíticas. La interpretación que Popper hace de Jenófanes se basa en la tesis de que sería redundante afirmar «supongamos que estas cosas son probables» (ver Popper, 1963, p. 236), sin embargo, este enunciado tendría sentido si Jenófanes estuviera usando una noción frecuentista de probabilidad. En la teoría de la verosimilitud, las afirmaciones sobre la probabilidad son tan falibles como lo puedan ser las afirmaciones sobre la verdad (ver Niiniluoto, 1987, cap. 7).

17 Uno de los puntos principales del «probabilismo» jesuítico de Bartolomé de Medina (final del siglo XVI) consistía en considerar moralmente lícito seguir una opinión probable, aunque la opinión opuesta sea más probable (ver Kantola, 1994, p. 126). Una conclusión similar se sigue de la apuesta de Pascal, que sopesa las pérdidas y las ganancias de la creencia y el descreimiento religioso (cf. Hacking, 1975). 


\section{VI.2. SEGUIR LAS APARIENCIAS}

En un trabajo famoso (pero, por lo que yo sé, todavía inédito) Myles Burnyeat defiende que "Carnéades no fue probabilista». No se trata sólo de la sospecha de que Carnéades no se comprometió con la doctrina de la probabilidad, como ya afirmó Pierre Couissin en 1929 (cf. Couissin, 1983), sino, lo que importa aún más, que pithanon connota «persuasivo» o «verdad aparente» más bien que «probabilidad» o «credibilidad» (cf. Burnyeat, 1983).

Para Burnyeat, lo esencial del escepticismo antiguo es la distinción entre apariencia y realidad. Tanto el escepticismo académico como el pirrónico defendieron una «vida sin creencia», en la que la epoche significa negar el asentimiento a todo lo que no esté dado en la apariencia. Sexto Empírico (1985, p. 40) expresó esta idea al decir que en la vida diaria no vivimos dogmáticamente, pero "prestamos la debida atención a las apariencias», siguiendo la «guía de la naturaleza, la compulsión de los sentimientos, la tradición de las leyes y costumbres y la enseñanza de las artes».

Según esta interpretación, verosimilitud significa «apariencia», «apariencia de verdad», e incluso «ilusorio». Esta connotación la encontramos en la palabra alemana para «probabilidad», Wahrscheinlichkeit, ya que Schein significa «apariencia».

El origen del término pithanon en la tradición de la argumentación retórica presta cierto apoyo a la interpretación de Burnyeat (cf. Glucker, 1995). También es relevante aquí el desarrollo posterior de la retórica y la poética (véase una detallada exposición en Mehtonen, 1996). En De Inventione, Cicerón distinguió tres tipos de narraciones: historia, argumentum y fabula. Mientras que la primera tiene como objetivo proporcionar una descripción verdadera de los eventos reales, y la tercera es pura ficción (e. g. las historias fantásticas de dragones), el segundo tipo de narración contiene ocurrencias que «podrían haber sucedido». Así, en el argumentum se incluyen, por ejemplo, las comedias «realistas» que describen personas y sucesos ficticios, pero que son «posiblemente verdaderos» en el sentido de que el relato satisface las expectativas de la opinión pública y de la audiencia. En el siglo primero de nuestra era, Horacio, en su Ars Poetica exigió a toda obra poética que fuera proxima veris (cercana a la verdad), y Quintiliano, en Institutio Oratoria, caracterizaba un argumentum como una narración vero simile. Dicho uso fue seguido en las teorías poéticas del siglo XII por autores como Teodorico de Chartres y Guillermo de Conches. Esta idea de verosimilitud se ha mantenido viva en las teorías modernas de la literatura (cf. Todorov, 1977). Es interesante observar que la palabra francesa vraisemblance (traducida como «verosimilitud» (verisimilitude) o «aparentemente real» (seeming-real) ha sido empleada desde los años sesenta en la teoría cinematográfica para expresar la 
naturaleza artificial o engañosa del cine en tanto que representación ilusoria (ver Andrew, 1984).

La vida sin creencias sobre una realidad que trascienda las apariencias no es una forma de realismo ingenuo antimetafísico. Seguir las apariencias es más bien la regla que gobierna lo que Jean-François Lyotard (1985) ha llamado la «condición postmoderna». ${ }^{18} \mathrm{De}$ acuerdo con esta interpretación del escepticismo antiguo, el pirronismo contemporáneo incluiría, además de a los postmodernistas franceses (los cuales hoy día siguen las apariencias producidas por las luces de neón), al anarquismo epistemológico de Paul Feyerabend (1987) y al neopragmatismo de Richard Rorty (1989), quien suspende el juicio sobre la metafísica y reclama solidaridad con las normas contingentes de su propia cultura local.

\section{VI.3. FIABILIDAD}

Aristóteles definió «probable» (gr. eoikota) como aquello que ocurre usualmente. La referencia directa a tales ideas estadísticas o frecuentistas parece ser muy escasa en las discusiones de los escépticos. Cabe mencionar, sin embargo, dos ejemplos interesantes.

Tras caracterizar las impresiones vívidas con apariencia de verdad, Carnéades señaló que «en general» esas impresiones son verdaderas (Bett, 1989), p. 72). Y aunque esto no es un criterio de creencia, es suficiente para la dirección de la vida. En otras palabras, las impresiones pithane son fiables para la acción práctica puesto que su frecuencia de verdad (en el sentido de Peirce y de Goldman) es alta.

Una idea similar se expresa en un importante ejemplo de San Agustín: cuando vemos un niño, no podemos saber si se parece a su padre a menos que lo conozcamos también a él.

Te ruego, dije, que me prestes toda tu atención. Si un hombre que nunca ha visto a tu padre, al ver a tu hermano afirmase que se parece a tu padre, ¿no pensarías que está trastornado mentalmente o hablando sin pensar? (San Agustín, 1950, p. 82).

Mas he aquí una de las respuestas del escéptico académico Licencio:

No necesariamente, replicó, a menos que insista en que sabe que eso es así. Obviamente, si lo entiende como probable ya que frecuentemente se dice que es así, no puede ser acusado de ligereza alguna. (Ibid., p. 84).

18 Ver también la explicación de Jean Baudrillard (1984) de los «simulacros» que en calidad de «hiperreales» encubren la desaparición de la realidad detrás de los signos y las imágenes. 
En otras palabras, si los hijos se parecen frecuentemente a sus padres, entonces podemos suponer con un grado de fiabilidad bastante alto que un hombre determinado guarda parecido con su padre.

\section{VI.4. VEROSIMILITUD}

¿Cabe imaginar que lo que los escépticos de la Academia querían decir con «probabilidad» es algo parecido al sentido contemporáneo de «verosimilitud»-en vez de probabilidad epistémica o frecuencia-de-verdad? En ese caso habrían sido falibilistas fuertes 1500 años antes que Nicolás de Cusa y 2000 años antes que Popper. Y la respuesta oficial de la Academia al argumento de la apraxia, o sea, la regla de seguir la verosimilitud, habría sido una versión temprana de la regla popperiana de la verosimilitud alta para el problema pragmático de la inducción.

En 1929 Couissin afirmó que «lo que se parece a la verdad» es «una frase sin sentido» (Couissin, 1983, p. 46). Todavía en 1960 Quine se mostraba de acuerdo con este veredicto. Por tanto, el intento de encontrar anticipaciones de un concepto semejante entre los filósofos antiguos puede parecer poco realista. Sin embargo, en otro lugar he mostrado que San Agustín formuló dicha idea, aunque sin apoyarla, en su diálogo con los escépticos (Niiniluoto, 1987, p. 161). A continuación argumentaré que Cicerón la había expuesto ya palabra por palabra.

En primer lugar, en un momento de la discusión, San Agustín explica la doctrina secreta de la Academia y da a entender que lo-que-es-como-la-verdad (what-is-like-truth), es decir, aquello en lo que «ellos veían una similitud admirable con la verdad», en realidad no es lo verdadero (San Agustín, 1950, p. 147)). Pero esta es precisamente la tesis del falibilismo fuerte, que el concepto de verosimilitud se aplica a enunciados falsos. ${ }^{19}$ Sin embargo, en una obra posterior San Agustín desaprueba esta formulación (Ibid., p. 193).

Volvamos, en segundo lugar, al ejemplo del hijo y del padre. Su misión era establecer que el escéptico no puede conocer lo-que-es-como-la-verdad a

19 En la tradición medieval de la poética, el concepto de verosimilitud fue usado también en un sentido parecido al del falibilismo fuerte. Una narración verosímil era tenida normalmente como falsa (en relación a los eventos que realmente ocurrieron), pero, al mismo tiempo, como cercana a la verdad (ver Mehtonen, 1996). Sin embargo, en la poética, la cuestión era recomendar modos de representación para las historias ficticias que las hicieran plausibles ante la audiencia, no sugerir que la verosimilitud podía servir como una base para la acción. 
no ser que conozca ya la verdad, y puesto que el escéptico niega esta última posibilidad, la anterior queda también excluida:

Es obvio que de la misma manera son ridículos tus académicos, puesto que dicen que en los asuntos prácticos ellos siguen «lo-que-es-como-la-verdad», aunque realmente no saben qué sea la verdad misma. (Ibid., p. 85).

Es importante notar que en este ejemplo aparece implicada una relación de similitud, que es el elemento clave del modo en que entiende la verosimilitud el enfoque de la similitud (Niiniluoto, 1987, cap. 1). Y la principal conclusión de San Agustín -que la verosimilitud no es conocida si no lo es la verdad-es una objeción habitual a las teorías de la verosimilitud existentes (ver ibid., p. 265).

Christopher Kirwan (1983), quien sitúa el origen de este ejemplo en el Fedón de Platón (74d9-e4), ${ }^{20}$ puntualiza que San Agustín no puede referirse a la ignorancia de lo que es la verdad o de lo que significa la verdad. Sin embargo, cree que se confunde el hecho de que una proposición sea parecida a alguna verdad en particular (lo que nos lleva al concepto de verosimilitud relativa a un objetivo determinado) con el hecho de que sea parecida a una verdad cualquiera (lo que equivale a la definición de verdad aproximada). Kirwan sostiene, frente a San Agustín, que la última cuestión podría ser resuelta si supiéramos "cómo sería una verdad en caso de que la hubiera», incluso aunque en realidad no existieran verdades, pero esta tesis no tiene análogo en la teoría contemporánea de la verosimilitud.

En tercer lugar, Cicerón utiliza muchas formulaciones que parecen indicar claramente la idea de una similitud y un parecido con la verdad o una distancia a la verdad. Por ejemplo:

Puesto que ellos dicen que puede sucederle al hombre sabio que, aun después de haber tomado todas las precauciones y analizado la cuestión del modo más cuidadoso, le surja algo que, asemejándose a la verdad, estuviera en realidad muy lejos de ella, serán incapaces de confiar en sí mismos, incluso aunque de todas formas avancen un largo trecho en el camino hacia la verdad real, como suelen decir, o se acerquen a ella todo lo posible. (II, xi).

En cuarto lugar, hay muchos pasajes en los que el escéptico expone la opinión característica del falibilista fuerte de que en la ciencia y en la filosofía la búsqueda de la verdad puede aproximarnos a la verdad sin alcanzarla

20 Se podrían traer también a colación las palabras de Jesús en el Evangelio de San Juan 14: 7-10: «El que me ha visto a mí ha visto al Padre». 
realmente. Uno de esos pasajes se encuentra en la discusión sobre la relación entre verdad y felicidad en Contra Academicos:

«¿Eres de la opinión de que podemos ser felices incluso aunque no hayamos encontrado la verdad?» Aquí Licencio interpuso: «Sí, si buscamos la verdad.» (San Agustín, 1950, p. 40).

(Licencio) «Dejame preguntarte, pues, ¿por qué no va a poder ser feliz un hombre que busca la verdad, incluso aunque nunca la encuentre?» «Porque», contestó Trigetio, «un hombre feliz, tal como lo imaginamos, ha de ser un hombre sabio, perfecto en todas las cosas. Mas alguien que está todavía buscando no es perfecto.» (Ibid., p. 42).

«Estoy de acuerdo», dijo él (Licencio), «en que alguien que no ha llegado a su meta no es perfecto. Pero en mi opinión sólo Dios, o quizás el alma del hombre una vez que ha abandonado su cuerpo, esa prisión oscura, puede conocer la verdad de la que hablamos. Sin embargo, la finalidad del hombre queda cumplida en la búsqueda de la verdad. En efecto, nuestro ideal es la perfección, pero una perfección propia del hombre.» «En tal caso», dijo Trigetio, «el hombre no puede ser feliz. ¿Cómo podría serlo, si no puede alcanzar lo que más profundamente desea? Pero el hecho es que la vida de un hombre puede ser feliz, dado que puede vivir de conformidad con la parte de su espíritu que verdaderamente debe gobernar al hombre. Por lo tanto, él puede encontrar la verdad.» (Ibid., p. 45-46). ${ }^{21}$

Cicerón cree también que la verosimilitud es el fin propio del hombre:

El único objeto de nuestras discusiones es argumentar desde ambas partes para trazar y dar forma a algunos resultados que puedan ser verdaderos o una aproximación lo más cercana posible a la verdad (...exprimant aliquid quod aut verum sit aut ad id quam proxime accedat) (Acad. II, iii).

Pues ¿cómo puedo dejar de ansiar el descubrimiento de la verdad, cuando me regocijo al descubrir algo que se parece a la verdad? ...Soy un hombre de opiniones (ya que no soy un hombre sabio). (II, xx).

[...] Mientras que si se nos ocurre una idea que parece guardar una similitud con la verdad, el espíritu se llena del placer más humanizador. Por tanto, estas

21 Entre los filósofos contemporáneos de la ciencia, Larry Laudan (1984) coincide con Trigetio en que no es racional pretender metas que no puedan ser alcanzadas. Sin embargo, Laudan también está de acuerdo con el escéptico en que la verdad es una meta utópica o inaccesible para la ciencia. Puesto que piensa además que las discusiones sobre la verosimilitud son «demasiada palabrería», concluye que no debe entenderse la ciencia como una actividad de búsqueda de la verdad. Es posible entender la versión del «pragmatismo» que ofrece Laudan como una variante de un falibilismo muy débil, ya que recomienda la acción sobre la base de la teoría mejor confirmada, pero la confirmación no es una señal de la verdad de la teoría misma, sino sólo de la verdad de las predicciones empíricas efectuadas desde ella. Esta postura es cercana a la idea carnapiana de confirmación por casos. Cf. la discusión en Niiniluoto (1984, 1997). 
investigaciones serán emprendidas tanto por vuestro hombre sabio como por el nuestro, si bien el vuestro tendrá la intención de asentir, creer y afirmar, mientras que el nuestro estará resuelto a ser cauto para no llegar a opiniones precipitadas y a darse por satisfecho en asuntos de esta índole si descubre aquello que guarda similitud con la verdad. (II, xli).

Aquí el académico escéptico está expresando la perspectiva que habitualmente consideramos como el espíritu de la Ilustración moderna: es más importante buscar el conocimiento que poseerlo. En palabras de Peirce, «no es el conocimiento, sino el amor al saber, lo que caracteriza al hombre de ciencia» (CP 1.44).

\section{CONCLUSIONES}

En la epistemología moderna, el falibilismo es una via media entre el dogmatismo y el escepticismo. Su origen histórico se encuentra en una rama de la antigua escuela del escepticismo académico. Ya que la diferencia entre las formas fuerte y débil del falibilismo, así como la distinción entre probabilidad epistémica y verosimilitud, sólo han sido comprendidas en las últimas dos décadas, no podemos esperar encontrar formulaciones claras de dichas doctrinas entre los filósofos griegos y romanos. Pero hemos mostrado que las dos ideas centrales del falibilismo - el grado de certeza y el grado de cercanía a la verdad- están presentes en la tradición escéptica al menos desde los tiempos de Cicerón.

\section{REFERENCIAS BIBLIOGRÁFICAS}

AGASSI, J. (1963) Towards a Historiography of Science, The Hague: Mouton. ANDREW, D. (1984) Concepts in Film Theory, Oxford: Oxford University Press. AGUSTINE, St. (1950) Against the Academics, Westminister: The Newman Press.

BAUDRILLARD, J. (1984) «The Precession of Simulacra», in B. Wallis (ed), Art After Modernism: Rethinking Representation, New York: The New Museun of Contemporary Art, pp. 253-281.

BETT, R. (1989) «Carneades' Pithanon: A Reappraisal of its Role and Status», in J. Annas (ed), Oxford Studies in Ancient Philosophy, Oxford: Oxford University Press, pp. 59-94.

BRADLEY, F. H. (1983) Appearance and Reality, London: George Allen.

BURNYEAT, M. (1980) «Can the Sceptic Live His Scepticism?», in Schofield et al., pp. 20-53. Reimpreso en Burnyeat (1983), pp. 117-148.

BURNYEAT, M. (ed) (1983) The Skeptical Tradition, Berkeley: University of California Press.

BYRNE, E. (1968) Probability and Opinion: A Study of Medieval Presuppositions of Post-Medieval Theories of Probability, The Hague: M. Nijhoff.

CARNAP, R. (1971) «Inductive Logic and Rational Decisions», in R. Carnap y R. 
Jeffrey (eds), Studies in Inductive Logic and Probability, vol. I, Berkeley: University of California Press, pp. 5-31.

CICERÓN (1956) De naura deorum, Academica, London: William Heinemann.

COPLESTON, F. (1966) A History of Philosophy, vol. I, London: Burns and Oates.

COUISSIN, P. (1983) «The Stoicism of the New Academy», in Burnyeat (1983), pp. 31-63.

CUSA, N. de (1954) Of Learned Ignorance, London: Routledge and Kegan Paul.

CHISHOLM, R. (1977) Theory of Knowledge, Englewood Cliffs: Prentice Hall.

DEWEY, J. (1960) The Quest for Certainty, New York: Capricorn Books.

DUHEM, P. (1969) To Save the Phenomena: An Essay on the Idea of a Physical Theory from Plato to Galileo, Chicago: The University of Chicago Press.

EWING, A. C. (1934) Idealism: A Critical Survey, Strand: Methuen.

FEYERABEND, P. (1984) «Xenophanes: A Foreruner of Critical Rationalism?», in G. Andersson (ed), Rationality in Science and Politics, Dordrecht: Reidel, pp. 95-109.

FEYERABEND, P. (1987) Farewell to Reason, London: Verso.

GARBER, D. y ZABELL, S. (1979) «On the Emergence of Probability», Archive for History of Exact Sciences 21, 33-53.

GLUCKER, J. (1995) «Probabile, Veri Simile, and Related Terms», in J.G.F. Powell (ed), Cicero the Philosopher, Oxford: Oxford University Press, pp. 115-143.

GOLdMAN, A. I. (1986) Epistemology and Cognition, Cambridge, Ma.: Harvard University Press.

HACKING, I. (1975), The Emergence of Probability, Cambridge: Cambridge University Press.

JAMES, W. (1956) The Will to Believe and Other Essays in Popular Philosophy, New York: Dover.

JARDINE, L. (1983) «Lorenzo Valla: Academic Skepticism and the New Humanist Dialectic», in Burnyeat (1983), pp. 253-286

JEFFREY, R. (1984) «De Finetti’s Probabilism», Synthese 60, 73-90.

KANT, I. (1974) Logic, Indianapolis: Bobbs-Merrill.

KANTOLA, I. (1994) Probability and Moral Uncertainty in Late Medieval and Early Modern Times, Helsinki: Luther-Agricola-Society.

KIRWAN, C. (1983) «Agustine against the Skeptics», in Burnyeat (1983), pp. 205224.

LAUDAN, L. (1973) «Peirce and the Trivialization of the Self-Corrective Thesis», in R. Giere and R. Westfall (ed), Foundations of Scientific Method: The Nineteenth Century, Bloomington: Indiana University Press, pp. 275-306.

LAUDAN, L. (1981) Science and hypothesis, Dordrecht: Reidel.

LAUDAN, L. (1984) Science and Values, Berkeley: University of California Press.

LEHRER, K. (1990) Theory of Kowledge (Boulder: Westview).

LEEUWEN, H. G. van (1970) The problem of Certainty in English Thought 16301690, Amsterdam: Nijhoff.

LEVI, I. (1967) Gambling With Truth, New York: Alfred A. Knopf.

LONG, A. A. (1986) Hellenistic Philosophers: Stoics, Epicureans, Sceptics, 2nd ed., Berkeley: University of California Press. 
LONG, A. A. y SEDLEY, D. N. (eds) (1988), The Hellenistic Philosophers, Cambridge: Cambridge University Press.

LYOTARD, J.-F. (1985) The Postmodern Condition: A Report on Knowledge, Minneapolis: University of Minnesota Press.

MEHTONEN, P. (1996) Old Concepts and New Poetics: Historia, Argumentum, and Fabula in the Twelfth- and Early Thirteenth-Century Latin Poetics of Fiction, Helsinki: Societas Scientiarum Fennica.

MUSGRAVE, A. (1993) Common Sense, Science and Scepticism, Cambridge: Cambridge University Press.

NIINILUOTO, I. (1977) «On the Truthlikeness of Generalizations» in R. E. Butts y J. Hintikka (eds), Basic Problems in Methodology and Linguistic, Logic, Methodology, and Philosophy of Science V, Part Three, Dordrecht: Reidel, pp. 121-147.

NIINILUOTO, I. (1978) «Notes on Popper as Follower of Whewell and Peirce», Ajatus 37, pp. 272-327. Reimpreso en Niiniluoto (1984), pp. 18-60.

NIINILUOTO, I. (1984) Is Science Progressive?, Dordrecht: Reidel.

NIINILUOTO, I. (1987) Truthlikeness, Dordrecht: Reidel.

NIINILUOTO, I. (1997) Critical Scientific Realism, (en prensa).

NIINILUOTO, I. y TUOMELA, R. (1973) Theoretical Concepts and HypotheticoInductive Inference, Dordrecht: Reidel.

ODDIE, G. (1986) Likeness to Truth, Dordrecht: Reidel.

PAPPAS, G y SWAIN, M. (eds) (1978) Essays on Knowledge and Justification, Ithaca: Cornell University Press.

PEIRCE, C. S. (1931-35) Collected Papers, vols. 1-6 ed. por C. Hartshorne y P. Weiss, Cambridge, MA.: Harvard University Press.

POPKIN, R. H. (1979) The History of Scepticism from Erasmus to Spinoza, Berkeley: University of California Press.

POPPER, K. R. (1963) Conjectures and Refutations, London: Routledge and Kegan Paul.

POPPER, K. R. (1979) Objective Knowledge, ed. rev., Oxford: Oxford University Press.

QUINE, W. v. O. (1960) Word and Object, Cambridge, MA.: The MIT Press.

RESCHER, N. (1973) The Coherence Theory of Truth, Oxford: Oxford University Press.

RORTY, R. (1984) «The Historiography of Philosophy: Four Genres», in R. Rorty, J. B. Schneewind y Q. Skinner (eds), Philosophy in History, Cambridge: Cambridge University Press, pp. 49-75.

RORTY, R. (1989) Contingency, Irony, and Solidarity, Cambridge: Cambridge University Press.

SCOFIELD, M., BURNYEAT, M. y BARNES, J. (eds) (1980) Doubt and Dogmatism: Studies in Hellenistic Epistemology, Oxford: Oxford University Press.

SELLARS, W. (1968) Science and Metaphysics, London: Routledge and Kegan Paul.

SEXTUS EMPIRICUS (1985) Selections from the Major Writings on Scepticism, Man, and God, ed. por P. P. Hallie, Indianapolis: Hackett. 
SIHVOLA, J. (1996) «Vakuuttavat vaikutelmat: antiikin skeptisismi ja tiedon mahdollisuus», in T. M. Holopainen y T. J. Holopainen (eds), Sielun liikkeitä, Helsinki: Gaudeamus, pp. 59-68.

SUPPES, P. (1981) «Limitations of the Axiomatic Method in Ancient Greek Mathematical Sciences», in J. Hintikka, D. Gruender y E. Agazzi (eds), Theory Change, Ancient Axiomatics, and Galileo's Methodology, Dordrecht: Reidel, pp. 197-213.

STRIKER, G. (1980) «Sceptical Strategies», in Scofield et al., pp. 54-83.

TODOROV, T. (1977) The Poetics of Prose, Oxford: Blackwell.

UNGER, P. (1975) Ignorance: A Case for Scepticism, Oxford: Oxford University Press.

VAN FRAASSEN. B. (1980) The Scientific Image, Oxford: Oxford University Press.

WATKINS, J. (1984) Science and Scepticism, Princenton: Princentom University Press.

IKKA NiIniLuOto es profesor de filosofía en la Universidad de Helsinki desde 1981. Actualmente es Profesor Emérito. Ha sido también rector de dicha universidad y miembro de la Academia de Finlandia.

Líneas de investigación:

Realismo científico, progreso científico, versoimilitud, ciencias de diseño

Obras principales:

(1984) Is Science Progressive?, Dordrecth / Boston: D. Reidel Publishing Company.

(1987) Truthlikeness, Dordrecth / Boston: D. Reidel Publishing Company.

(1999) Critical Scientific Realism, Oxford and New York: Oxford University Press.

(2018), Truth-Seeking by Abduction, Cham (Switzerland): Springer. 